\title{
Alfvén wave coupled with flow-driven fluid instability in interpenetrating plasmas
}

\author{
J. Vranjes ${ }^{1}$ \\ Instituto de Astrofisica de Canarias, 38205 La Laguna, Tenerife, Spain and \\ Departamento de Astrofisica, Universidad de La Laguna, 38205 La Laguna, Tenerife, \\ Spain.
}

(Dated: 3 December 2021)

The Alfvén wave is analyzed in case of one quasineutral plasma propagating with some constant speed $v_{0}$ through another static quasineutral plasma. A dispersion equation is derived describing the Alfvén wave coupled with the flow driven mode $\omega=k v_{0}$ and solutions are discussed analytically and numerically. The usual solutions for two oppositely propagating Alfvén waves are substantially modified due to the flowing plasma. More profound is modification of the solution propagating in the negative direction with respect to the magnetic field and the plasma flow. For a large enough flow speed (exceeding the Alfvén speed in the static plasma), this negative solution may become non-propagating, with frequency equal to zero. In this case it represents a spatial variation of the electromagnetic field. For greater flow speed it becomes a forward mode, and it may merge with the positive one. This merging of the two modes represents the starting point for a flow-driven instability, with two complex-conjugate solutions. The Alfvén wave in interpenetrating plasmas is thus modified and coupled with the flow-driven mode and this coupled mode is shown to be growing when the flow speed is large enough. The energy for the instability is macroscopic kinetic energy of the flowing plasma. The dynamics of plasma particles caused by such a coupled wave still remains similar to the ordinary Alfvén wave. This means that well-known stochastic heating by the Alfvén wave may work, and this should additionally support the potential role of the Alfvén wave in the coronal heating.

PACS numbers: 52.35.Bj, 52.30.-q, 96.60.-j

\section{INTRODUCTION}

Alfvén wave (AW) has been a hot topic in plasma physics ever since its discovery ${ }^{1}$, both in the general plasma theory and laboratory plasmas 211 , and in astrophysics 12 14. This is even more so for solar atmosphere which is a perfect natural laboratory for various plasma waves and instabilities, and almost any of the plasma modes observed in the laboratory or predicted by theory may be expected and/or observed there.

The Alfvén wave is widely believed to play an important role in the heating of upper layers in the solar atmosphere. Indeed, the wave has apparently been detected in numerous observations $\sqrt{15 / 16}$, and it has been studied in a great amount of works in the past (to mention just a few like for example $e^{17}(21)$. In fact this is by far the most studied mode in the solar plasma environment.

In view of its potential role in the coronal heating, and this even at frequencies far below the particle gyrofrequency, known as non-resonant (stochastic) heating 21 23, it is of great importance to search for reliable sources for the the AW and to be able to distinguish it from some other electromagnetic (EM) perturbations and instabilities which may exhibit similar behavior.

One of such EM instabilities develops when one flowing (f) plasma with its specific set of parameters propagates through another static (s) plasma whose parameters may in principle be different. Both plasmas separately may be quasi-neutral, or the quasi-neutrality applies for the system as a whole (both situations have been studied in the literature) and they may have different densities $n_{f}$ and $n_{s}$. The indices $f, s$ will be used to denote parameters in the two respective plasmas.

Within kinetic theory, in our recent papers ${ }^{924}$, we have shown that a new kind of instability may develop in such systems. The instability discussed for longitudinal electrostatic perturbations is purely kinetic by nature and current-less (the latter meaning that the plasma contains no currents in the initial state). For such modes, the real part of the wave frequency is mainly determined by the static plasma parameters, while the instability is mainly due to ions from the flowing plasma, and these features make it very different from classic currentcarrying plasmas where the instability is caused by electrons passing through static ions.

When one population of particles moves as a whole through a plasma, there may be a collective mode associated with this motion 27 , and an instability may develop 28 even in fluid theory, and both real and imaginary parts of the wave frequency may be completely determined by the flow. The properties of such flow-driven (FD) instabilities and modes may sometimes be similar to normal plasma waves, and some care is needed to make a clear distinction between them.

In the present work we shall describe some features of an electromagnetic FD instability coupled with the AW within fluid theory, and consequences of this coupling on the AW. In such a system of two mutually coupled plasmas (one flowing and another static) the usual AW cannot be expected in any of the two plasmas separately. Instead, it will be shown that a coupled mode develops with some properties (e.g., dynamics of particles involved 


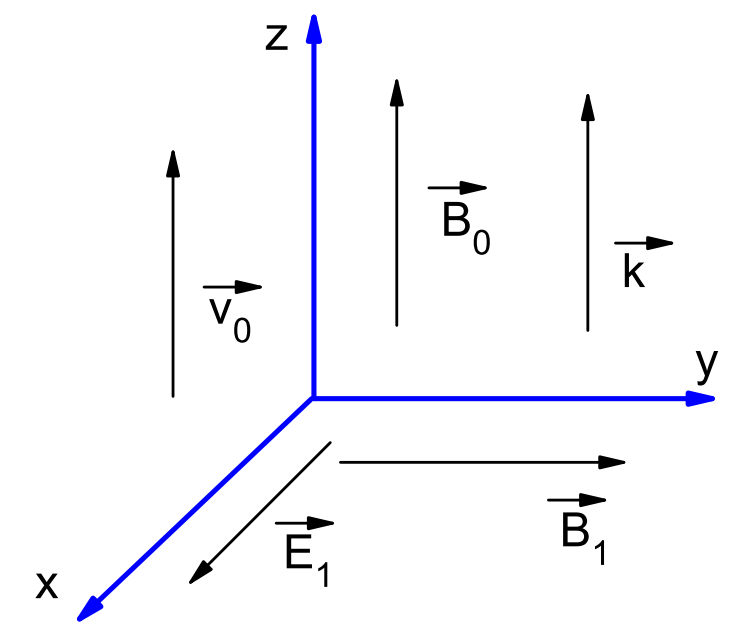

FIG. 1. Geometry of the EM wave propagating in the direction of the magnetic field.

in the wave) similar to the usual AW, but the usual AW may become modified by the flow even for relatively small flow speed. For a fast enough flow the resulting mode may be unstable and completely determined by the flow, and yet (for some specific cases of the plasma flow) the wave frequency may still be exactly equal to the usual AW frequency of the static plasma (see later in the text and Figs. 2 3), although in most cases it is different.

Unaware of the presence of the flowing plasma, and using the usual AW as a tool for remote diagnostics, observers might come out with rather inaccurate estimates regarding the values of local plasma parameters. The purpose of this work is to point out what may be expected in such coupled plasmas and how to interpret observations appropriately.

\section{BASIC EQUATIONS AND MODEL}

We shall assume one flowing (f) electron-ion plasma moving with the speed $\vec{v}_{0}=v_{0} \vec{e}_{z}$ through another static (s) electron-ion plasma plasma, and we shall use standard equations appropriate for electromagnetic perturbations. Each of the plasmas is initially separately currentless. The flow is in the direction of a background magnetic field $\vec{B}_{0}=B_{0} \vec{e}_{z}$, see Fig. 1 .

The perturbations are in the low-frequency limit $\omega \ll$ $\Omega_{i}=e B_{0} / m_{i}$, the ion mass in both plasmas is the same and for the two systems we have separate quasineutrality conditions satisfied $n_{f i 0}=n_{f e 0}=n_{f 0}, n_{s i 0}=n_{s e 0}=$ $n_{s 0}$. In such a regime the perturbations may be assumed as linearly polarized ${ }^{29}$ and we may assume that the perturbed magnetic field is $\vec{B}_{1}=B_{y} \vec{e}_{y}$, similar to the case of an ordinary AW. Perturbations are assumed of the shape $\sim \exp (-i \omega t+i k z)$ and we use the usual MHD equations for the two plasmas. From the Faraday law we have

$$
\vec{E}_{1}=\frac{\omega B_{y}}{k} \vec{e}_{x}
$$

We further use Ohm's law and omit all unessential terms like electron mass corrections, collisions, and Hall effect (details about the latter can be found elesewhere ${ }^{30}$ ), and for the static plasma we have $\vec{E}_{1}+\vec{u}_{1} \times \vec{B}_{0}=0$, which yields

$$
\vec{u}_{1}=-\frac{\vec{B}_{0} \times \vec{E}_{1}}{B_{0}^{2}}=-\frac{\omega}{k} \frac{B_{y}}{B_{0}} \vec{e}_{y}
$$

For the flowing plasma we have $\vec{E}_{1}+\vec{v}_{1} \times \vec{B}_{0}+\vec{v}_{0} \times \vec{B}_{1}=0$, which yields

$$
\vec{v}_{1}=-\frac{\vec{B}_{0} \times \vec{E}_{1}}{B_{0}^{2}}+\frac{v_{0}}{B_{0}} \vec{B}_{1}=-\frac{\omega}{k} \frac{B_{y}}{B_{0}} \vec{e}_{y}+v_{0} \frac{B_{y}}{B_{0}} \vec{e}_{y} .
$$

The Ampère law for the present case of the two plasmas reads:

$$
\nabla \times \vec{B}=\mu_{0}\left(\vec{j}_{s}+\vec{j}_{f}\right) .
$$

The currents $\vec{j}_{s, f}$ can be calculated from the MHD momentum equations

$$
\begin{gathered}
\rho_{s} \frac{\partial \vec{u}_{1}}{\partial t}=\vec{j}_{s 1} \times \vec{B}_{0}, \\
\rho_{f}\left(\frac{\partial}{\partial t}+\vec{v}_{0} \cdot \nabla\right) \vec{v}_{1}=\vec{j}_{f 1} \times \vec{B}_{0},
\end{gathered}
$$

where $\rho_{j}=\left(m_{e}+m_{i}\right) n_{j}$. This yields the dispersion equation for low frequency EM perturbations propagating in the direction of the magnetic field

$$
\omega^{2}\left(1+\frac{v_{A f}^{2}}{v_{A S}^{2}}\right)-2 k v_{0} \omega+k^{2}\left(v_{0}^{2}-v_{A f}^{2}\right)=0 .
$$

Here $v_{A j}^{2}=B_{0}^{2} /\left(\mu_{0} \rho_{j}\right)$ are the Alfvén speeds for the two separate plasmas. Eq. (6) describes coupled AW and flow driven (FD) mode. The frequency of the propagating perturbations is

$$
\frac{\omega}{k}=\frac{1}{1+n_{s} / n_{f}}\left\{v_{0} \pm v_{A s}\left[\frac{n_{s}}{n_{f}}\left(1+\frac{n_{s}}{n_{f}}-\frac{v_{0}^{2}}{v_{A s}^{2}}\right)\right]^{1 / 2}\right\} .
$$

Further in the text, the two solutions with plus and minus signs will be called $\mathrm{P}$ and $\mathrm{N}$ solutions, respectively. Quite generally, there can be no instability as long as $v_{0} \leq v_{A s}$. However, there may be an instability on condition

$$
v_{0}^{2}>v_{A S}^{2}\left(1+\frac{n_{s}}{n_{f}}\right)=v_{A S}^{2}+v_{A f}^{2} .
$$

From Eq. (7) it can also be concluded that the instability is absent if the density of the flowing plasma is small, i.e., if the following condition is satisfied:

$$
\frac{n_{f}}{n_{s}} \leq \frac{1}{v_{0}^{2} / v_{A s}^{2}-1} .
$$

The features of the growing solutions are discussed further in the text. 


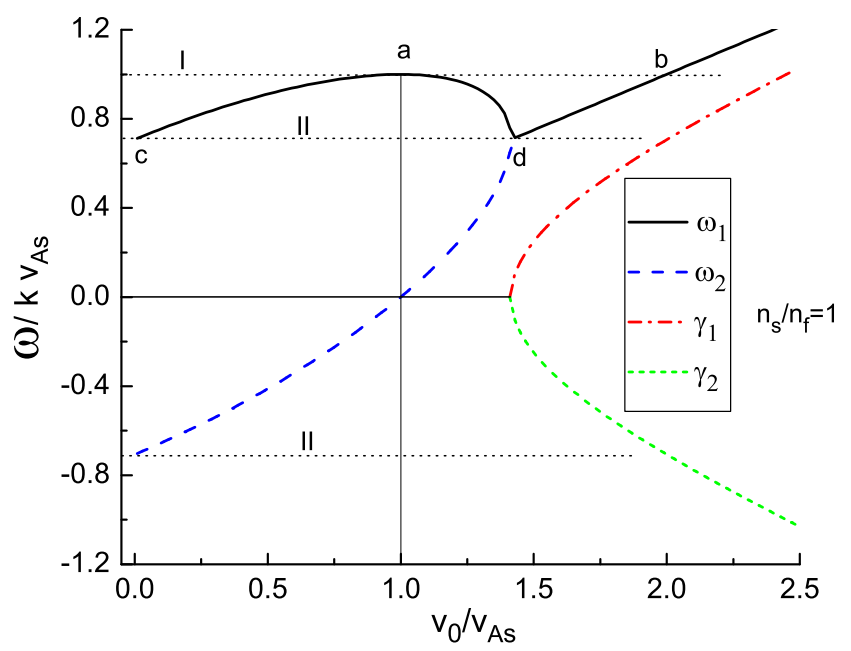

FIG. 2. Two solutions given by Eq. (7) for two interpenetrating plasmas of the same density: normalized frequency in terms of the normalized speed of the flowing plasma.

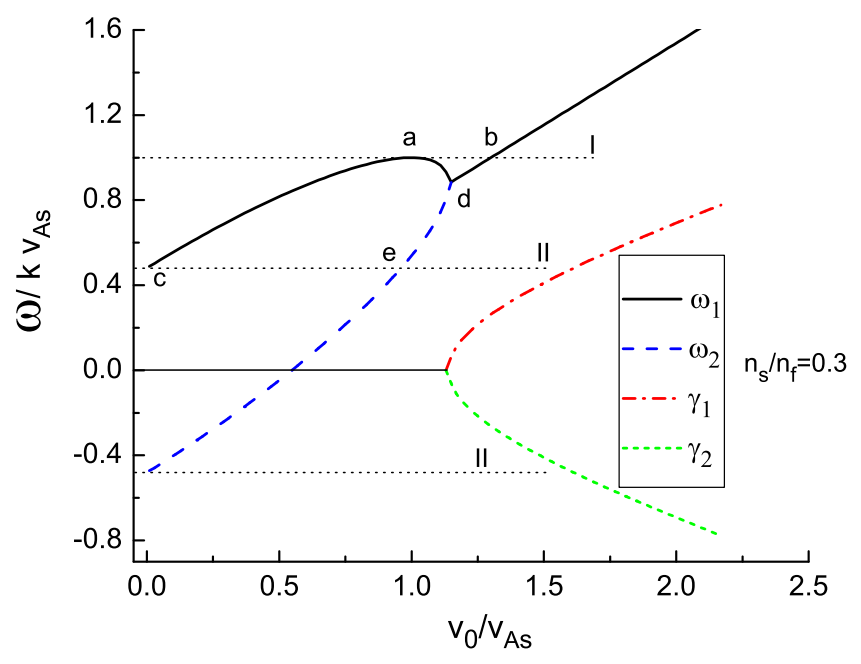

FIG. 3. The same as in Fig. 2 but for $n_{s} / n_{f}=0.3$.

\section{PROPERTIES OF SOLUTIONS IN THE PRESENCE OF FLOWING PLASMA}

Without the flowing plasma, from Eq. (7) we have the $\mathrm{AW}$ in the static plasma $\omega_{s}=k v_{A s}$. In the presence of both plasmas but with a negligible flow we have $\omega_{s+f}=B_{0} /\left[\mu_{0} m\left(n_{s}+n_{f}\right)\right]^{1 / 2}$. These two characteristic limits are presented by dotted lines I, II in Figs. 2, 3. Only in these particular limits one may speak about the ordinary AW, the normalized frequency is constant, hence the horizontal lines I, II. In the figures, for the densities $n_{s} / n_{f}=1,0.3$, respectively, the line II has values $0.71,0.48$, and this combined AW frequency $\omega_{s+f}$ is marked by the letter $c$ in figures.

However, for any finite value of $v_{0}$, the wave frequency is modified by the flow. For the two density ratios $n_{s} / n_{f}$, the solution becomes complex, $\omega=\omega_{r}+i \gamma$, for $v_{0}>v_{A s}$

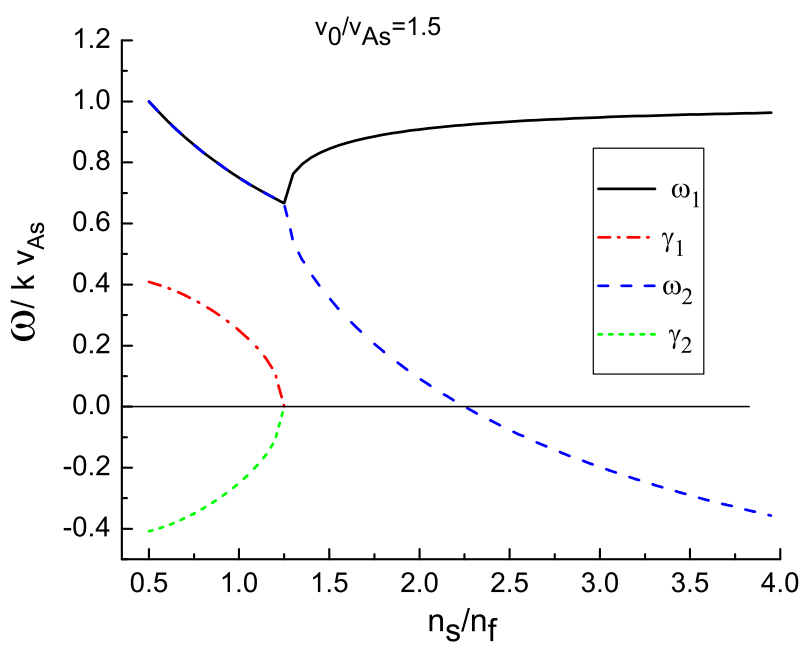

FIG. 4. Vanishing of instability for reduced density of the flowing plasma, and transformation of the $\mathrm{N}$ solution from negative to forward, in terms of the density ratio of two plasmas.

greater than 1.41 and 1.15 respectively, for parameters in Figs. 2, 3, as can be concluded from Eq. (8), i.e., the threshold is reduced for a more dense flowing plasma. This is logical as it implies more energy stored in the flow. It is interesting to observe that in the range of the stable mode $(\gamma=0)$ the real frequency for the P-solution in combined two plasmas may become equal to the AW frequency in static plasma $\omega_{s}$; this is a direct consequence of the flow due to which the frequency is increased up to the line I. One of these situations is marked by letter $a$ in Figs. $2,3$.

Note that in this same case the frequency for the Nsolution for parameters in Fig. 2 passes through zero. This implies that there is no propagation, and we have just a spatial variation of the magnetic field, with the spatial length $2 \pi / k$. In case when the flowing plasma is heavier, this point of passing through zero frequency is shifted towards smaller values of $v_{0}$ as Fig. 3 shows. It is remarkable that in general, the N-solution becomes a forward mode and it can have practically any frequency in the range from $-\omega_{s+f}$ up to nearly $\omega_{s}$ as can be concluded from Figs. 2, 3, Hence, if flow effects are not taken into account properly, there exists an obvious possibility for misidentification of modes and for mistakes in estimate of plasma parameters when wave characteristics are used for remote plasma probing. Further, the P- and $\mathrm{N}$-solutions become coupled when the latter becomes a forward wave and when it is increased enough, and this is the reason for decreasing line of $\mathrm{P}$-solution in the range $a-d$.

From quadratic dispersion equation (6) it is easily seen that two identical real solutions exist (denoted by letter $d$ in Figs. 2, 3) whenever

$$
v_{0}=\left(v_{A S}^{2}+v_{A f}^{2}\right)^{1 / 2}=v_{A S}\left(1+n_{s} / n_{f}\right)^{1 / 2}
$$


This point represent the place where $\mathrm{P}$ and $\mathrm{N}$ solutions merge. Also, whenever the densities of the two interpenetrating plasmas are equal, the frequency of the resulting flow-modified mode at this merging point $d$ equals $\omega_{s+f}$. But this is only for $n_{s} / n_{f}=1$, observe that in Fig. 3 the point $d$ is off the line II. The point $e$ introduced in Fig. 3 denotes the place where $\mathrm{N}$-solution line (which is also a forward mode in this domain of $v_{0}$ ) crosses the line II.

Equally peculiar is the frequency marked by letter $b$, which is in the unstable mode regime when there is only one pair of complex-conjugate solutions. In that range both real and imaginary parts of the frequency are determined by the flow, yet the real frequency part is equal to $\omega_{s}$. This means that the solution can easily be misidentified as the usual AW although clearly in this case one would have no explanation for the wave growth. So a possibility for misidentification of modes exists in both stable and unstable limits and for both $\mathrm{P}$ and $\mathrm{N}$ solutions; those are the stable point $a$ and unstable $b$ and they are relevant to AW $\omega_{s}$, and those are all points of the N-mode when its frequency is positive (including the stable point $d$ ).

The features related to condition $(9)$ are presented in Fig. 4. For a fixed value $v_{0}$ the density ratio $n_{s} / n_{f}$ in the graph is allowed to change and the instability vanishes for small enough flowing plasma density $n_{f}$; in the present case this is for $n_{f}<0.8 n_{s}$. Thus the instability preferably develops when a more dense plasma is accelerated into a dilute one. The $\mathrm{N}$ solution becomes forward $\omega_{2}>0$ at around $n_{f}=0.44 n_{s}$.

Finally, it should be stressed that for any given wavenumber $k$ we may have several possible flow speeds that give exactly the same real part of the wave-frequency. In Fig. 2 any real part of the P-frequency $\omega_{1}$ between lines I and II may be obtained for no less than three different values of $v_{0}$ (with one of the three cases being unstable), while in Fig. 3 this is narrowed to frequencies above those corresponding to the point $d$ and below line I. On the other hand, in Fig. 4 it is seen that the same real frequency $\omega_{1}$ may be obtained (for any $k$ ) for two different values of the ratio $n_{s} / n_{f}$. So there is an obvious ambiguity in determining plasma parameters through the wave frequency.

\section{DISCUSSIONS}

When one plasma enters another plasma and propagates through it, an electromagnetic Alfvénic instability may develop if the speed of the flowing plasma exceeds the Alfvén speed of the static plasma, and this appears as a necessary instability condition. The supply energy for the instability is the kinetic energy of the streaming plasma. We have demonstrated that the instability may develop only when the density of the streaming plasma exceeds some critical value. The sufficient instability conditions are given by Eqs. (8, 9).

The AW behavior in the presence of a flowing (with speed $v_{0}$ ) plasma can be summarized as follows: a) as long as $v_{0}^{2} / v_{A s}^{2}<1+n_{s} / n_{f}$ there are two modified solutions for the AW, one is always positive $(\mathrm{P})$ and the other is negative $(\mathrm{N})$ for small $v_{0}, \mathrm{~b}$ ) due to the flow the $\mathrm{N}$ solution may propagate in the positive direction (note that we keep it calling $\mathrm{N}$ solution although it becomes positive, only in order to distinguish it from the $\mathrm{P}$ solution) and for $v_{0}^{2} / v_{A S}^{2}=1+n_{s} / n_{f}$ the two solutions merge, c) for $v_{0}^{2} / v_{A s}^{2}>1+n_{s} / n_{f}$ there are two complex-conjugate solutions.

In general, more profound effects of the flow are on the $\mathrm{N}$ solution, as may be concluded from Figs. 2, 3 The $\mathrm{P}$ solution phase speed increases till it reaches $v_{A s}$, but for a greater flow speed it decreases (the region between the points $a$ and $d$ in Figs. 2, 3). This happens in the range $1+\epsilon<v_{0}^{2} / v_{A s}^{2}<1+n_{s} / n_{f}$ where $\epsilon$ can be any number in the range $0<\epsilon<n_{s} / n_{f}$.

Quite generally, the instability is expected to work well in an environment with relatively weak magnetic field. In fast expanding astrophysical clouds (which are typically more dense than the surrounding interstellar space) the instability should be ubiquitous.

Throughout the text, frequency and growth rate are calculated normalized to AW frequency in the static plasma $k v_{A s}$ so the conclusions are general and valid for any wavelength. However, for frequencies in the range of ion gyro-frequency or even higher, circular polarization should be taken into account. Such a generalization and coupling with ordinary and extraordinary EM waves can easily be done.

Various effects may to some extent modify (or reduce) the instability, like viscosity which can easily be included and it will consequently affect the wave mainly at shorter wavelengths ${ }^{10}$, and friction. In the corona itself the ionion collisions are $\operatorname{rare}^{31}$ and the collision frequency is around $0.07 \mathrm{~Hz}$. In the case of one ion species coming from lower layers and colliding with coronal ions, the corresponding expressions for the collision frequency in such a situation are available in the literature ${ }^{32}$, and the collision frequency is increased to a few Hz. However, the instability discussed here can clearly be very strong and these effects are not expected to play a major role.

Important points to discuss are: i) how realistic the mode and instability discussed here are, ii) how likely it is that this mode is misidentified in observations as an ordinary AW and what the consequences of this could be, and, iii) if abundant, how important this mode may be, in particular for heating in the solar plasma environment.

i) Examples of interpenetrating plasmas in space and astrophysics are numerous, like colliding astrophysical clouds, and plasmas originating from the explosions of novae and supernovae and moving through the surrounding interstellar space.

An obvious example of such plasmas is seen also in the solar atmosphere. Observations ${ }^{33134}$ describe jets generated in the transition region of the solar atmosphere with the rate of about 24 events per second throughout the solar atmosphere, and having a few thousand kilometers in 
diameter. Their upward speed was around $400 \mathrm{~km} / \mathrm{s}$. Up-flows of plasma in spicules may reach any height in the corona between $5 \cdot 10^{3} \mathrm{~km}$ and $2 \cdot 10^{5} \mathrm{~km}$, with diameters sometimes greater than $1000 \mathrm{~km}$. They appear to be ubiquitous, $10^{5}$ events at any time and covering a few percent of the solar atmosphere. An opposite phenomenon, called the coronal rain belongs to the same sort of phenomena $\sqrt{34}$, where plasma performs almost a free-fall speed between 50 and $100 \mathrm{~km} / \mathrm{s}$. So interpenetraing/permeating plasmas appear to be a rather common phenomena in space plasmas.

Regarding the point ii), the real part of the obtained frequency shows some peculiar features: for two critical values of the flow speed $v_{0}$, the frequency of P-solution (true forward mode) becomes the same as the AW frequency $\omega_{s}$ in the static plasma (the points denoted as $a$ and $b$ in Figs. 2, 3). For yet another value of $v_{0}$ and for equal densities of the two plasmas, the wave frequency becomes equal to the AW frequency of the two plasmas combined $\omega_{s+f}$ (the point $d$ in Fig. 2). The second (Nsolution) is with negative frequency only in the domain of small flow speed, while for greater speed $v_{0}$ it is a forward mode as well. With such features it fits neither into the usual AW theory (where only one forward mode with the given polarization may exist), nor into the usual flowdriven mode $\omega=k v_{0}$, because the flow can only produce one (forward) mode, which is the P-solution discussed above. So the forward propagating N-solution is an intrinsic result of coupling between AW and FD modes, but it may have a positive frequency (including values close to $\omega_{s}$ ) and it can be misidentified as an ordinary AW whenever its frequency is positive. This implies that observers might deduce incorrect values for local plasma parameters by assuming it is an ordinary AW.

From practical side, rather important is the situation described by the point $b$ because it describes a growing wave which should easily develop and can consequently be observed. On the other hand, from Eqs. (1, 2) it may be seen that the fluid motion in the presence of the flowing plasma (the term with $v_{0}$ ) is in the same direction as in the case of an ordinary AW. The transverse displacement is of the same magnitude as long as $v_{0}$ is of the same order as the phase speed. Thus, both frequency and the fluid displacement may be completely similar to the AW case (although clearly in this regime this is more the FD mode). The awareness of the existence of this coupled AW-FD mode wave is thus of essential importance.

Regarding the issue iii), the AW and FD modes are obviously closely interrelated. The resulting coupled mode has a clearly identified source, which is not always so for the standard AW. The features of both modes are similar, so quite generally, eventual presence of this electromagnetic flow-driven instability in the solar plasma environment can only strengthen the role of the AW in the process of heating. According to the numbers on solar spicules 16 , the speeds in spicules are up to 100 $\mathrm{km} / \mathrm{s}$, but the Alfvén speed $v_{A s}$ is always too big to have the instability. Even taking a rather weak field of $10 \mathrm{G}$ and (too) high density $n_{s}=10^{17} \mathrm{~m}^{-3}$ yields AW speed around $70 \mathrm{~km} / \mathrm{s}$, so the instability may work only in regions with weak field, and it could thus contribute to heating preferably in regions outside of strong magnetic structures where a proper model for heating is yet to be established. However, the situation may be rather different in case of the mentioned jets 33 where the speed $v_{0}$ is far greater, and the magnetic field may in principle be of the value given above or weaker. In view of large spatial scales of these jets, the electromagnetic instability presented here may develop on quite large scales in horizontal direction, after a jet reaches altitudes at which the conditions 8, 9) become satisfied. So the total volumes within which the growing modes develop may be rather substantial.

The result presented in the work are relevant to numerous studies on the non-resonant stochastic heating of plasmas $21 \sqrt{23}$ by AW. In these studies this stochastic mechanism of heating has been discussed specifically in application to stellar atmospheres. The heating has various features that may explain observations, like better heating of heavier particles, better heating in the perpendicular direction with respect to the magnetic field, acceleration of ions in the parallel direction, and the obtained heating time ${ }^{21}$ is of the order of $\pi /\left(k v_{T i}\right)$.

In application to the solar corona, for wavelengths of 1 and $100 \mathrm{~km}$ this gives heating times for protons roughly of the order of $1 / 200 \mathrm{~s}$ and $1 / 2 \mathrm{~s}$, respectively. The obtained temperature increase ${ }^{21}$ in parallel and perpendicular direction for protons was by factor 3.2 and 15, respectively, and the achieved anisotropy was around $T_{\perp} / T_{\|} \simeq 5$. Protons are in average accelerated to the speed of around 0.15 Alfvén speed. The anisotropy obtained for $\mathrm{O}^{5+}$ ions was 10-18. They estimated the heating time for $\mathrm{O}^{5+}$ ions would be 10-40 minutes in the solar corona for waves with 2 minutes wave-period. The reported heating time ${ }^{22}$ was just a few gyro-rotation periods. It is pointed out that a significant level of AW is needed for theory to be realistic.

In the present work we have shown that plasma dynamics in interpenetrating plasmas remains similar to the ordinary AW case, while in the same time we provide a realistic energy source for such an AW heating. In other words, the mentioned condition about the required AW level is satisfied by the fact that we indeed have a growing mode. This all implies that the energy of the flowing plasma can directly be transformed into heat through this stochastic heating mechanism.

\footnotetext{
${ }^{1}$ H. Alfvén, Nature 150, 405 (1942).

${ }^{2}$ T. K. Allen, W. R. Baker, R. V. Pyle, and J. M. Wilcox, Phys. Rev. Lett. 2, 383 (1959).

${ }^{3}$ A. Barnes, Phys. Fluids 9, 1483 (1966).

${ }^{4}$ M. F. Bashir, Z. Iqbal, I. Aslam, and G. Murtaza, Phys. Plasmas 17, 102112 (2010).

${ }^{5}$ A. Gigliotti, W. Gekelman, P. Pribyl, S. Vincena, A. Karavaev, X. Shao, A. S. Sharma, and D. Papadopoulos, Phys. Plasmas 16 092106 (2009).

${ }^{6}$ J. V. Hollweg, Phys. Rev. Lett. 27, 1349 (1971).

${ }^{7}$ M. N. Rosenbluth and P. H. Rutherford, Phys. Rev. Lett. 34, 1428 (1975).

${ }^{8}$ B. S. Tanenbaum and D. Mintzer, Phys. Fluids 5, 1226 (1962).
} 
${ }^{9}$ J. Vranjes and M. Kono, Phys. Plasmas 21, 042104 (2014). ${ }^{10} \mathrm{~J}$. Vranjes, MNRAS 445, 1614 (2014).

${ }^{11}$ C. J. Watts and J. Hanna, Phys. Plasmas 11, 1358 (2004).

${ }^{12}$ A. Gazol and T. Passot, Astron. Astrophys. 411, 1 (2003).

${ }^{13}$ R. Kulsrud and W. P. Pearce, Astrophys. J. 156, 445 (1969).

${ }^{14}$ R. E. Pudritz, Astrophys. J. 350, 195 (1990).

${ }^{15}$ D. B. Jess, M. Mathioudakis, R. Erdelyi, P. J. Crockett, F. P. Keenan, and D. J. Christian, Science 323, 1582 (2009).

${ }^{16}$ B. De Pontieu, S. W. McIntosh, M. Carlsson, V. H. Hansteen, T. D. Tarbell, P. Boerner, J. Martinez-Sykora, C. J. Schrijver, and A. M. Title, Science 331, 55 (2011).

${ }^{17}$ L. Fletcher and H. S. Hudson, Astrophys. J. 675, 1645 (2008).

18 J. V. Hollweg, Solar Phys. 56, 305 (1978).

${ }^{19}$ J. V. Hollweg, Solar Phys. 70, 25 (1981).

${ }^{20}$ K. Muerawski and Z. E. Musielak, Astron. Astrophys. 518, A37 (2010).

${ }^{21}$ Q. Lu and X. Li, Phys. Plasmas 14, 042303 (2007).

${ }^{22}$ C. B. Wang, C. S. Wu, and P. H. Yoon, Phys. Rev. Lett. 96,
125001 (2006)

${ }^{23}$ C. S. Wu and P. H. Yoon, Phys. Rev. Lett. 99, 075001 (2007).

${ }^{24}$ J. Vranjes, S. Poedts, and Z. Ehsan, Phys. Plasmas 16, 074501 (2009).

${ }^{25}$ J. Vranjes, Phys. Plasmas 18, 084501 (2011).

${ }^{26}$ J. Vranjes, Astron. Astrophys. 554, A90 (2013).

${ }^{27}$ S. Ichimaru, Basic Principles of Plasma Physics (The Benjamin/Cummings Pub. Co., Reading, 1973), p. 144.

${ }^{28}$ V. P. Dokuchaev, Sov. Phys. J. Exp. Theor. Phys. 12, 292 (1961).

${ }^{29}$ F. F. Chen, Introduction to Plasma Physics and Controlled Fusion (Plenum Press, New York, 1988).

${ }^{30}$ B. P. Pandey and M. Wardle, MNRAS 426, 1436 (2012).

${ }^{31}$ J. Vranjes, Astron. Astrophys. 458, 635 (2006).

${ }^{32}$ J. Vranjes, M. Kono, S. Poedts, and M. Y. Tanaka, Phys. Plasmas 15, 092107 (2008).

${ }^{33}$ G. E. Brueckner and J. D. F. Bartoe, Astrophys. J. 272, 329 (1983).

${ }^{34}$ A. O. Benz, Plasma Astrophysics: Kinetic Processes in Solar and Stellar Coronae (Kluwer, Dordrecht, 2002), p. 7. 